\title{
VII. - ACTION DES ANTIBIOTIQUES SUR PORCELETS ALLAITÉS
}

\author{
PAR
}

E. SALMON LEGAGNEUR, et M. MICHEL

Station de Recherches sur l'Élevage et Service de Biochimie

Centre National de Recherches Zootechniques, Jouy en Josas

\section{PLAN DU MEMOIRE}

\section{Introduction.}

I. - Protocole expérimental.
A. - Antibiotiques utilisés.
B. - 'Technique de distribution.
C. - Animaux.
D. - Dosage des antibiotiques.
E. - Etude des flores intestinales.

\section{II. - Résultats.}

A. - Consommation d'antibiotiques.
a) dans la terre ;
b) dans le lait.

B. - Croissance des porcelets :

a) poids au sevrage ;

b) courbes de croissance.

C. - État sanitaire des portées :
a) mortalité ;
b) diarrhées;
c) régularité.

D. - Etude des flores intestinales :

a) Examen microscopique ;

b) Influence des antibiotiques sur les désaminases bactériennes.

\section{III. - Discussion. Conclusion.}

Avec la collaboration technique de Mue M-N. QuercLe. 


\section{INTRODUCTION}

Les études récentes de M. MicheL et A. Françors (I955) ont montré qu'il fallait probablement attribuer l'action des antibiotiques sur la croissance des animaux dù à une modification des propriétés biochimiques de la flore bactérienne du tractus intestinal. On peut expliquer ainsi pourquoi, dans le cas du porc, les meilleurs résultats enregistrés aient été obtenus avec une distribution des antibiotiques par voie buccale.

Or, dans le cas du porcelet allaité, chez qui l'ingestion des antibiotiques peut être extrêmement efficace du fait de la fragilité du jeune animal et de la fréquence des troubles intestinaux, ce mode de distribution n'avait pu être utilisé. En effet, le porcelet consomme à peu près exclusivement le lait maternel, jusqu'à l'âge de 3 semaines.

Différentes autres méthodes avaient donc été expérimentées, avec des résultats divers, par les chercheurs. Citons :

- l'implantation sous-cutanée de comprimés d'antibioliques, par Noland, (r952), Clawson, (r953), et VAchel et FÉvrier, (I954),

- l'injection intra-musculaire d'une solution huileuse de pénicilline par OLDFIELD, (I953),

- la distribution, par l'ïntermédiaire de la truie, en mélangeant les antibiotiques à la ration de la truie en gestation : NORD́FELDT, (I952), et BEEson, (I954), ou en lactation : CATron, (I953).

Seule par contre s'était avérée efficace, jusqu'à présent, l'incorporation d'antibiotiques (terramycine, auréomycine, pénicilline) à des laits synthétiques distribués à des porcelets élevés artificiellement, de la naissance au sevrage: (LUTHER (I952), CATRON (I952). Maiscette méthode, outre qu'elle s'éloigne sensiblement des conditions normales d'élevage, a conduit souvent à des résultats contradictoires dûs probablement à ce que la flore intestinale du porcelet est largement influencée par la nature du régime alimentaire distribué.

C'est ainsi par exemple, que D. Catron, (1952) et H. Schendel, (1954) estiment que les antibiotiques (terramycine et rimocidine) augmentent le nombre total des microorganismes, alors que pour WALHSTROM, (I952), les antibiotiques (auréomycine et chloromycétine) seraient sans action sur les bactéries du tube digestif, et en particulier sur les organismes du type clostridia et coli.

Le problème se posait donc de trouver une technique permettant l'ingestion aisée d'antibiotiques par les porcelets allaités naturellement.

Une observation courante montre que, dès les premiers jours après sa naissance, le porcelet est avide de terre qu'il parait consommer. L'idée nous est venue d'utiliser la terre comme support des antibiotiques que l'on désirait faire ingérer dès le premier âge. 
Des mesures préalables sur 4 portées groupant 35 porcelets nous ont confirmé cette observation et montré que la consommation de terre renouvelée fréquemment ( 3 fois par semaine) était en moyenne la suivante :

g. de terre fraîche par porcelet
et par jour

L'objet de la présente expérience a donc été, en partant de cette idée :

$r^{0}$ de mettre au point une méthode de distribution par voie orale d'antibiotiques à des porcelets allaités naturellement ;

$2^{0}$ d'étudier dans ces conditions l'influence d'un mélange d'antibiotiques sur la croissance et l'état sanitaire (réduction des diarrhées) des porcelets ;

$3^{\circ}$ d'obtenir des renseignements sur le mode d'action de ces antibiotiques, et plus particulièrement sur leur rôle sur la flore microbienne intestinale $\mathrm{du}$ porcelet.

\section{I. - PROTOCOLE EXPÉRIMENTAL}

\section{A. - Antibiotiques utilisés}

Nous avons utilisé un mélange d'auréomycine et de chloramphénicol, espérant obtenir un effet sur la croissance des animaux à la fois par action directe (auréomycine) et par réduction du "niveau de maladie", en particulier par une diminution des diarrhées du porcelet fréquentes à l'âge de $\mathrm{I}$ mois (chloramphénicol).

L'étude systématique des flores microbiennes isolées des excréments des animaux devait permettre de suivre les modifications entraînées par les antibiotiques, à l'état normal comme dans le cas de diarrhées, et d'établir cas échéant la laision pouvant exister entre ces deux phénomènes.

\section{B. - Technique de distribution}

La concentration d'antibiotique recherchée était celle des taux d'utilisation habituelle, soit to à $20 \mathrm{mcg}$ par centimètre cube de contenu intestinal, ce qui correspond à une teneur résiduelle de 3 à Io mcg par $\mathrm{g}$ de fecès humide. Compte tenu du volume du contenu intestinal aux différents âges, il fallait, pour obtenir ces valeurs, provoquer une ingestion quotidienne de Io mcg d'antibiotique chez le porcelet de 8 à I5 jours, et 20-30 mcg chez le porcelet d'un mois. 
a) De la naissance à I mois, le mélange d'antibiotiques était distribué dans de la terre.

L'incorporation des antibiotiques à la terre se faisait dans les conditions suivantes :

I $g$ d'auréomycine et I $g$ de chloramphénicol étaient mélangés à $200 \mathrm{~g}$ de terre séchée et pulvérisée. Cette terre était ensuite incorporée à $2,800 \mathrm{~kg}$ de terre fraîche et constituait ainsi la ration d'une portée pour deux jours.

Les témoins recevaient la même quantité de terre fraîche et celle-ci était changée trois fois par semaine.

b) De I mois à 2 mois, date du sevrage, la consommation de terre devenant difficilement contrôlable et les animaux s'alimentant plus régulièrement, les antibiotiques étaient distribués dans du lait écrémé, à raison de $0,05 \mathrm{~g}$ du mélange à parties égales d'antibiotiques par litre de lait.

Les consommations moyennes de lait écrémé avaient été évaluées à :

0,500 litre par porclet et par jour, à I mois
0,750 litre

c) Quelques jours avant le sevrage, les antibiotiques étaient progressivement supprimés de la ration.

\section{c. - Animaux}

L'expérience a porté sur $\mathrm{I} 6$ portées de porcs de race Large White, groupant au total $\mathrm{I} 28$ porcelets.

Les portées étaient associées par 2 (l'une recevant les antibiotiques; l'autre servant de témoin), compte tenu d'un certain nombre de critères : origine, âge, production laitière et résultats antérieurs de la truie, origine du père.

A l'intérieur des couples ainsi formés, les traitements étaient répartis au hasard.

A partir de 3 semaines, les porcelets recevaient, à volonté, un aliment complémentaire granulé contenant environ $150 \mathrm{~g}$ de matière protéique digestible par $\mathrm{kg}$ et du lait écrémé. Puis, à partir de la $\eta^{\mathbf{e}}$ semaine, deux repas humides du même aliment.

\section{D. - Dosage des antibiotiques}

Les fecès sont récoltés dès leur émission et traités de suite par 8 volumes d'acétone acide ( $\mathrm{pH} \mathrm{r}, 5)$. A près filtration, le pH est amené à 4,5, puis la solution est concentrée sous vide, filtrée et diluée à concentration convenable dans du tampon de $\mathrm{pH} 4,5$.

La méthode de dosage est celle indiquée dans une étude précédente (FÉvrier, Vachel et Michel, I954). 


\section{E. - Étude des ilores intestinales}

La flore microbienne des fecès est séparée des débris alimentaires par centrifugation fractionnée, puis lavée et diluée avec du sérum physiologique.

Sur chaque échantillon, on opère un examen microscopique après coloration par la méthode de Gram, et éventuellement un isolement de diverses espèces.

L'étude de certaines propriétés biochimiques de la flore, telle que son action désaminante, est effectuée sur la flore totale (MichEL, et Françors, I955.)

\section{II. - RÉSULTATS}

\section{A. - Consommation d'antibiotiques}

\section{a) de 1 à 30 jours (antibiotiques dans la terre)}

I8 échantillons moyens de fecès provenant des portées traitées ont été prélevés à différents stades de l'expérience. La teneur moyenne en antibiotique de ces échantillons a été de :

$3,32 \pm 1,42$ mcg par cc. de fecès humide.

Ce chiffre est très satisfaisant et correspond sensiblement aux résultats escomptés. De plus, l'écart type relativement faible montre que la méthode était fidèle et que l'absorption des antibiotiques par les porcelets a été très régulière ${ }^{(1)}$.

L'analyse de 5 échantillons de fecès de porcelets ayant eu la diarrhée a révélé une teneur moyenne en antibiotiques de 3,05. Ce chiffre n'est pas significativement différent du précédent.

Ces cas de diarrhées ne semblent donc pas pouvoir s'expliquer par une absence d'ingestion des antibiotiques.

\section{b) de 30 à 60 jours (antibiotiques dans le lait écrémé)}

5 échantillons seulement ont pu être analysés. Leur teneur en antibiotiques était :

I0,5 $\pm 3,03 \mathrm{mcg}$ par $\mathrm{cc}$. de fecès humide.

Cette valeur est sensiblement plus élevée que celle obtenue dans le cas

(I) Le rapport $\frac{\text { écart arithmétique moyen }}{\text { écart type }}$ est ici de 0,75 , ce qui caractérise une distribution très proche de la normale $(r=0,79)$. 
de la terre et dépasse un peu les prévisions. Il semble d'ailleurs que les porcelets absorbent des quantités assez variables de lait écrémé, ce qui entraîne une ingestion d'antibiotiques moins régulière avec le lait qu'avec la terre.

\section{B. - Croissance des porcelets}

\section{a) Poids au sevrage}

Le poids moyen à 2 mois des porcelets recevant des antibiotiques (65 animaux) a été de $20,55 \mathrm{~kg}$ contre $17,52 \mathrm{~kg}$ pour les porcelets témoins (63 animaux), ce qui correspond à des gains moyens journaliers, entre la naissance et le sevrage, de $3 \mathrm{I} 7 \mathrm{~g}$ dans le premier cas contre $268 \mathrm{~g}$ dans l'autre.

On trouvera au tableau I les poids moyens des porcelets des différentes portées.

La comparaison des résultats des deux groupes d'animaux fait ressortir une différence de poids au sevrage significative au seuil $\mathrm{P}=0,02$ $(t=3,06 \mathrm{I}$ pour $n=8)$.

TABLEAU I

Poids moyen des porcelets au sevrage

$\begin{array}{cc}\begin{array}{c}\text { Portées recevant } \\ \text { des antibiotiques }\end{array} \\ \text { des truies } & \begin{array}{c}\text { Poids des } \\ \text { porcelets }\end{array} \\ - & - \\ 2 \mathrm{~L} & \mathrm{~kg} \\ 887 & 23,288 \\ 970 & 25, \mathrm{I} 20 \\ 870 & 20, \mathrm{II} 8 \\ 722 & 19,618 \\ \text { I.43 } & 17,54 \mathrm{I} \\ \text { II } 7 \mathrm{I} & 20,800 \\ 868 & 18,750 \\ & \mathrm{I} 9,400\end{array}$

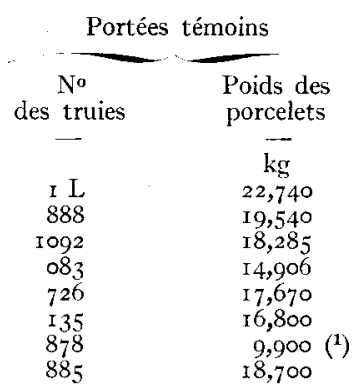

Différence moyenne entre poids au sevrage : $3,4 \mathrm{I} 7 \mathrm{~kg}$

\section{b) Courbe de croissance}

On trouvera au tableau II et à la figure I, les éléments de la croissance des deux groupes de porcelets entre la naissance et le sevrage.

(1) Le cas de cette portée aberrante pour une cause inexpliquée, ne modifie pas la valeur de l'expérience. L'élimination de cette portée et de son témoin donnerait un poids moyen au sevrage de $20,668 \mathrm{~kg}$ pour les porcelets ayant reçu des antibiotiques et de $18,149 \mathrm{~kg}$ pour les autres, soit une différence de $2,519 \mathrm{~kg}$. 


\section{TABLEAU II}

Poids moyen des porcelets aux différents âges (en $\mathrm{kg}$ )

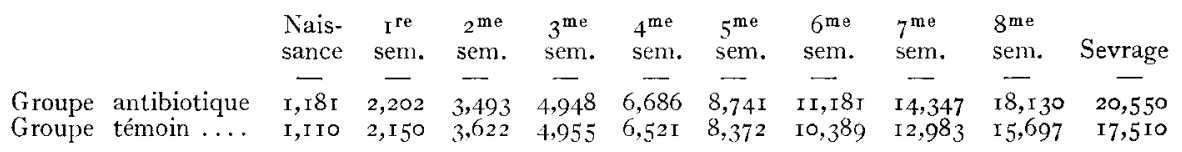

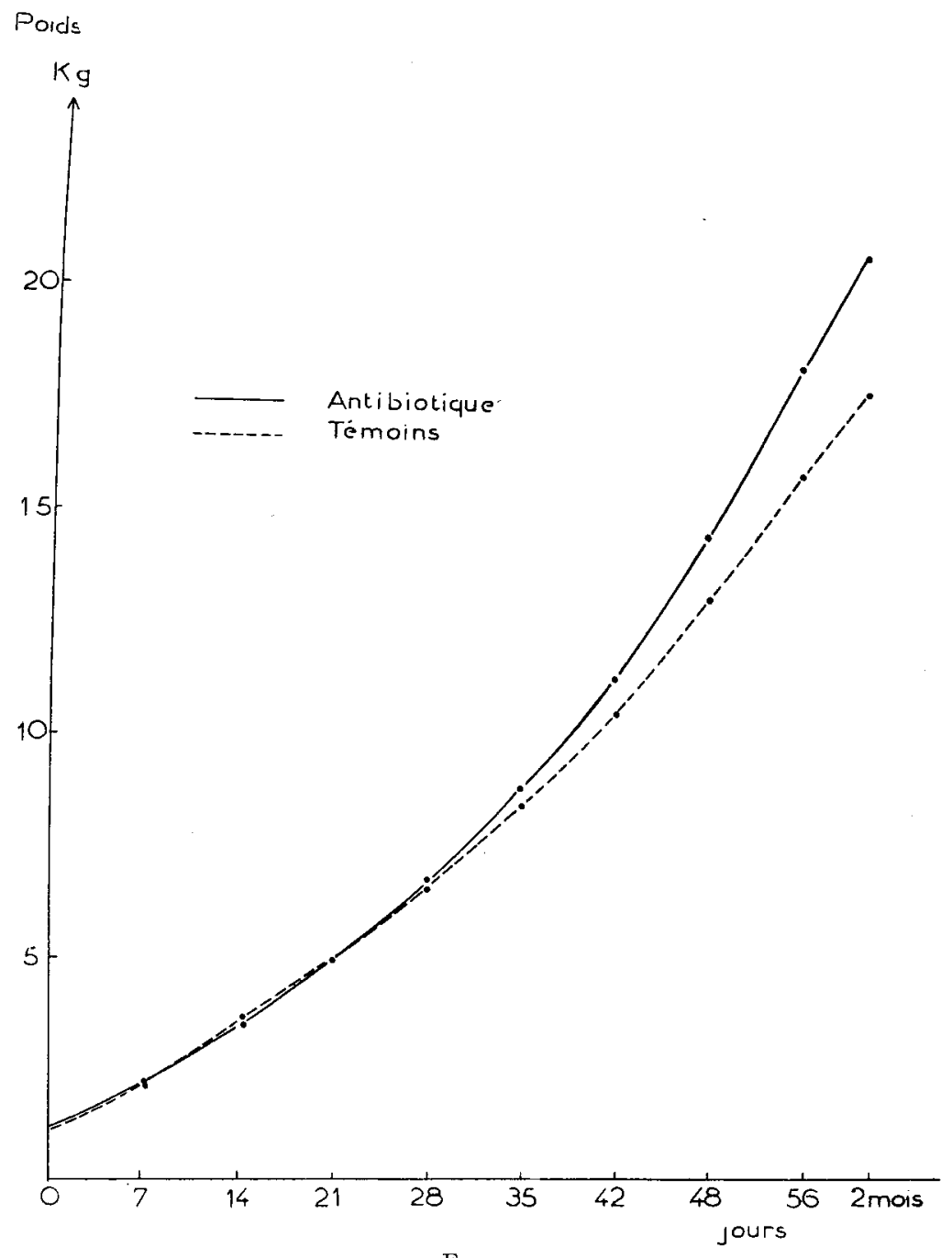

FIG, I

On notera en particulier le décrochage qui se produit entre les deux courbes à partir de la $3^{\mathrm{e}}$ semaine et qui va en augmentant jusqu'au sevrage. 
On remarquera, en outre, que la courbe des porcelets témoins accuse un léger fléchissement $\mathrm{d} u \mathrm{I}_{4}^{\mathrm{e}}$ au $28^{\mathrm{e}}$ jour, alors que celle des porcelets recevant antibiotiques, reste parfaitement soutenue pendantcette période.

\section{C. - État sanitaire}

\section{a) Mortalité}

La comparaison du nombre de porcelets nés et sevrés dans les deux -égimes fait apparaitre les chiffres suivants :

\section{TABLEAU III}

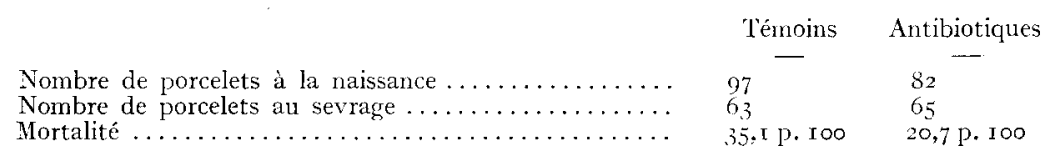

\section{b) Diarrhées}

Nous n'avons tenu compte ici que des cas de diarrhée survenus au moins 5 à 6 jours après la mise-bas. Les quelques cas de diarrhée plus précoces rencontrés ont pu être considérés comme normaux et faisant suite au meconium.

Le décompte des cas de diarrhée survenus entre la I ere semaine et le sevrage montre que 39 porcelets du régime témoin (soit environ 50 p. Ioo) ont été affectés (au moins passagèrement), contre $I 7$ porcelets dans le cas du régime antibiotique.

Si l'on ne retient que les cas de diarrhée persistantes (ayant duré 48 heures et plus), vraisemblablement d'origine microbienne, on trouve alors que I7 porcelets ont été malades dans le régime témoin contre 5 seulement dans le régime antibiotique.

La comparaison de ces chiffres montre donc une diminution marquée du nombre de cas de diarrhées pour les portées ayant reçu des antibiotiques.

\section{c) Régularité des portées}

Ce caractère est intéressant dans la mesure où l'on peut admettre qu'une portée où les porcelets n'ont pas souffert doit rester homogène.

En comparant la dispersion des poids au sevrage autour des moyennes des portées, on peut caractériser les 2 groupes d'animaux par les écarts types suivants : 
- pour les antibiotiques $s A= \pm I 6,068$ (exprimé en p. Ioo de la moyenne) moyenne.

- pour les témoins $\mathrm{s}^{\prime} \mathrm{T}= \pm \mathrm{I} 8,05 \mathrm{I}$ (exprimé en p. Ioo de la

I1 semblerait que les portées traitées atux antibiotiques aient été un peu plus régulières. Cependant, l'analyse de variance montre que la différence observée entre les deux chiffres précédents n'est pas significative :

$$
\left(\frac{\mathrm{s}^{\prime} \mathrm{T}-\mathrm{sA}}{\mathrm{s}(\mathrm{T}-\mathrm{A})}=0,927\right)
$$

Il est à noter cependant qu'il n'y a eu aucun " culot de portée " parmi les porcelets recevant des antibiotiques, alors qu'il y en a eu 3 parmi les porcelets du groupe témoin.

\section{1). - Étude des flores intestinales}

\section{a) Examen microscopique}

Les examens microscopiques directs montrent d'assez grandes variations d'un animal à l'autre, et chez un même animal, des variations en fonction des phénomènes digestifs.

L'ingestion des antibiotiques, aux doses indiquées, modifie assez peu le faciès microbien dans les fecès normaux d'animaux sains. On observe seulement, dans certaines portées, une augmentation des bacilles du genre sphérophorus, qui se retrouvent uniformément chez les individus qui croissent le plus vite.

Les différences les plus sensibles sous l'action des antibiotiques s'observent en cas de diarrhée. En présence d'antibiotiques, la flore est peu modifiée, sauf une légère augmentation des clostridia. Au contraire, chez les témoins, on trouve une disparition des streptobacilles Gram positifs, avec augmentation corrélative des colibacilles, staphylocoques et des streptocoques Gram négatifs.

I.es clostridia se retrouvent constamment en cas de diarrhée.

\section{b) Influence de l'ingestion d'antibiotique sur les désaminases microbiennes}

Les chiffres suivants indiquent les pourcentages moyens de dégradation de l'arginine par la flore fécale du porcelet:

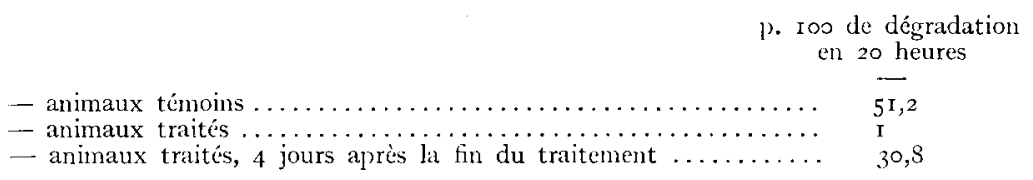


Ce blocage de la désamination confirme les résultats obtenus par ailleurs sur des porcs de roo $\mathrm{kg}$. Une semaine après la fin du traitement, la flore recouvre son pouvoir désaminant.

\section{III. - DISCUSSION. CONCLUSION}

La méthode de distribution d'antibiotiques par la terre, puis par le lait écrêmé à des porcelets allaités, semble avoir donné ici des résultats satisfaisants.

L'action conjuguée de 1'auréomycine et du chloramphénicol distribués aux porcelets de la naissance au sevrage, a permis d'enregistrer une augmentation significative $d u$ poids moyen des animaux au sevrage $(+3 \mathrm{~kg})$, en même temps qu'une certaine amélioration de l'état sanitaire et une diminution de la mortalité.

L'effet favorable du mélange d'antibiotiques utilisé peut être 1a conséquence du maintien d'une flore intestinale normale et du blocage de certaines de ses propriétés biochimiques. On retrouve ici la liaison, déjà constatée précédemment,par M. MICHEL, et A. Françors, entre le blocage des désaminases mesuré in vitro et l'augmentation de la vitesse de croissance sous l'action des antibiotiques.

Il ne semble pas, par ailleurs, que les antibiotiques aient eu ici une action déterminante sur l'importance et la physionomie des flores intestinales des porcelets sains. Il s'est en outre avéré, comme l'avaient déjà remarqué WALHSTROM et al., que ce mélange d'antibiotiques était incapable de réduire le nombre des bactéries du type clostridium, particulièrement importantes dans le cas de diarrhées. L'influence de l'antibiotique sur les propriétés biochimiques de la flore semble donc être la cause de l'augmentation de croissance des porcelets constatée ici.

La question n'est pas encore tranchée de savoir si l'abaissement du taux d'ammoniac passant dans la veine porte est responsable du meilleur état de santé des animaux ou si, corrélativement, c'est le taux des toxines microbiennes qui est abaissé (en effet selon HILLs, I940, les espèces désaminant l'arginine sont pathogènes).

Une étude ultérieure sera donc nécessaire pour apporter une solution aux problèmes biochimiques soulevés ici et, le cas échéant, améliorer de nouveau la croissance des animaux par l'emploi d'un antibiotique agissant spécifiquement sur les espèces pathogènes rencontrées.

\section{RÉFÉRENCES BIBLIOGRAPHIQUES}

Beeson (W. M.), Conred (J. H.), Andrews (F. N.). - The effect of different levels of aureomycin on the birth weight and livability of new born pigs. Mimeo AH 134, Purdue Univ., I954. 
Catron (D.), MADDOck. - Concentration of chlorotetracycline in sow's blood and milk after oral administration. Antibiot. et Chemotherapy, vol. 111, II nov. I953.

Catron (D.), cité par VACHEL. - Antibiotics in swine nutrition. Proc. of the, 4 ih Research Cont. Amer. Med. Inst., pp. I2-2I, I952.

Clawson (A. J.), SheFfy (B. E.), Willman (S. P.). - The value of implanted antibiotic pellet for suckling pigs. J. Anim. Sci., 12 (4), grI, I953.

FÉVRIER (R.), VAchel (J. P.), Michel (M.). - Les antibiotiques et la croissance du porc. A paraître aux Annales de Zootechnie, I954.

Hilis (G. M.). - Biochem. J., 34, I057. I940.

LUTHER (A. C.), Brown (J. H.). - Sows milk substitute for practical feeding of baby pig effects of various levels of antibiotics, fat... J. Anim. Sci., 10 (4), 772, I952.

Michei (M.), FrançoIs (A.). - Relation entre 1'influence des antibiotiques sur la croissance du porc et l'inhibition des désaminases de la flore intestinale. C. R. Acad. Sci., 240, 808, I955.

Noland (P. R.), TUCKER (R. L.). - Subcutaneous implantation of bacitracin in pellet to stimulate growth of suckling pigs. Arkansas $A g r$. Exp. Sta. Rpt., I952.

NORDFEIDT (S.). - Experiments with antibiotics and vitamin BI2 for pregnant and lactating sows with litters. Cantmanen $n^{\circ} 41$, I952.

OLDFIED (J. E.), HALE (O. M.). - A comparison of methods of administration of penicillin to suckling pigs. J. Anim. Sci., 11 (4), r952.

SCHENDEL (H. E.), BORG (A. F.), JoHNSON. - The effect of antibiotics on the intestinal and cecal microflora of baby pigs fed a synthetic milk ». J. Anim. Sci., 13 (4); 904, I954.

VACHEL, (J. P.), FÉVRIER (R.). - Les antibiotiques dans l'alimentation animale. Annales de Zootechnie, 1, 20, I953.

Wahlstrom (R. C.), Cohn (E. M.), Terrill (S. W.). - Growth effect of various antibiotics on baby pigs fed synthetic rations. I. Anim. Sci., 11. (3), 449, 1952. 